\title{
Esophagus Detection for Halal Classification in SYCUT
}

\author{
Nor Aziah Amirah Nor Muhammad, Rubiyah Yusof*, Reza Arfa, \\ Ridzuan Yunus, Nordinah Ismail
}

Centre for Artificial Intelligence \& Robotics (CAIRO), Universiti Teknologi Malaysia, 54100 Kuala Lumpur, Malaysia.Malaysia-Japan International Institute of Technology, Universiti Teknologi Malaysia, 54100 Kuala Lumpur, Malaysia

*Corresponding Author

DOI: https://doi.org/10.30880/ijie.2019.11.04.015

Received 25 April 2019; Accepted 1 August 2019; Available online 5 September 2019

\begin{abstract}
According to the Islamic Law, one of the procedures in halal slaughtering of chicken is the step of severing the trachea, esophagus and both the carotid arteries and jugular veins to accelerate the chicken's bleeding and death. Syariah Compliance Automated Chicken Processing System (SYCUT) uses the Vision Inspection Technology to detect and classify whether a chicken is halal or not. The lack of quality and halal assurance in chicken processing industry made it a need to produce such technology. The system implements image processing techniques and artificial intelligence approach, particularly the Viola and Jones object detection framework for esophagus detection. The results of the experiment from two different sites (Az-Zain and 3P) are 81.8\% and 55\% respectively. The detection module of those two sites show results of $95.6 \%$ and $93.5 \%$ which are the accuracy as good as human personnel.
\end{abstract}

Keywords: Chicken poultry, esophagus detection, halal, object detection, vision

\section{Introduction}

Object detection is widely used and an ongoing research in computer vision. The halal food industry in Malaysia requires the halal authentication by authorized bodies to perform inspection according to halal standards. However, due to the limitations of site visits by authorized body, there might be practices of non-syariah methods at the food processing sites (Selamat, 2011).

As mentioned in the paper by Normalina Omar \& Harlina Jaafar (2011), the word halal is an Arabic word which is lawful or permissible by Islamic laws. The use of halal expression in Malaysia is referred to the Trade Descriptions 1975 that can be applied to food. There are terms like 'Ditanggung halal' or 'Makanan halal' which indicates that a Muslim can consume the products as permitted by Islam. The term halalan toyyiban means any consumption of products that are not harmful and are safe to be consumed as underlined in the Syariah law, and thus is allowable and permissible (Mohd. Yunus, Wan Chik, \& Mohamad, 2010).

Normalina \& Harlina also states that the study found three pertinent areas that need to be focused on the halal food supply chain which are halal animal feed, proper slaughtering and proper segregation. Department of Islamic Development Malaysia (JAKIM) enforces and perform regular checks to ensure that the practices of the slaughtering process in the slaughterhouse are syariah compliance. There are a few requirements of the slaughtering process according to Syariah. This includes that the slaughter person must be a Muslim, use sharp knife to slaughter and the Tasmiyyah is recited and other factors, have to be followed by those who own the slaughterhouses (al-Qaradawi, 2013). The owner should also be concerned of the slaughterhouse's quality and cleanliness.

According to Yusof F. M. (2006), there are some advantages and moral aspects of Islamic way of animal slaughtering. This way of slaughtering can secure the consumer's health. This is because the blood which contains 
dirtiness, infections and diseases gushes out and drained from the slaughtered animal (Chand, 1995). This makes the meat that is separated from the impurities safe to be consumed without harm to the human body.

It is mentioned in Technofund Technical Report that a Syariah-Compliance Automated Chicken Processing System (SYCUT) has been developed to monitor the slaughtering process of the chicken to ensure that the chickens are slaughtered according to syariah and that the chickens are dead before the internal organs are removed and boiled. In this way, it is to confirm that the chicken is dead due to it being slaughtered and not because of being boiled or removal of internal organs (Selamat, 2011). This paper describes the methods of image processing and artificial intelligence techniques for the halal and non-halal chicken classification used by SYCUT.

According to the Islamic Law, one of the procedures in halal slaughtering of chicken is the step of severing the trachea (halqum), esophagus (mari') and both the carotid arteries and jugular veins (wadajain) to accelerate the chicken's bleeding and death (Department of Islamic Development Malaysia (JAKIM), 2011). Fig. 1 \& Fig. 2 show the anatomy of chicken's neck (Selamat, 2011).

In this paper, we presented the methods used for vision inspection system of the slaughtering process, specifically focussing on the detection of the esophagus which is the indication that the chicken is slaughtered properly according to the syariah. Various stages of image processing and image detection are performed in order to improve the accuracy of the system. The occlusion, illuminations and noises due to splattering of blood are some of the challenges of the systems which affect the accuracy.

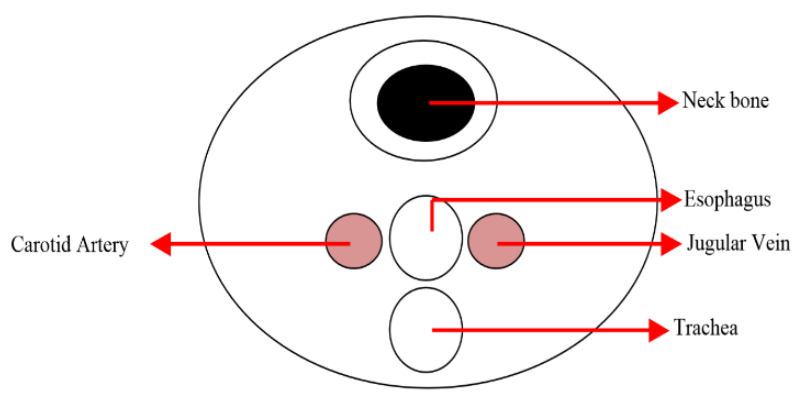

Fig. 1 - Cross section of chicken's neck (Selamat, 2011)

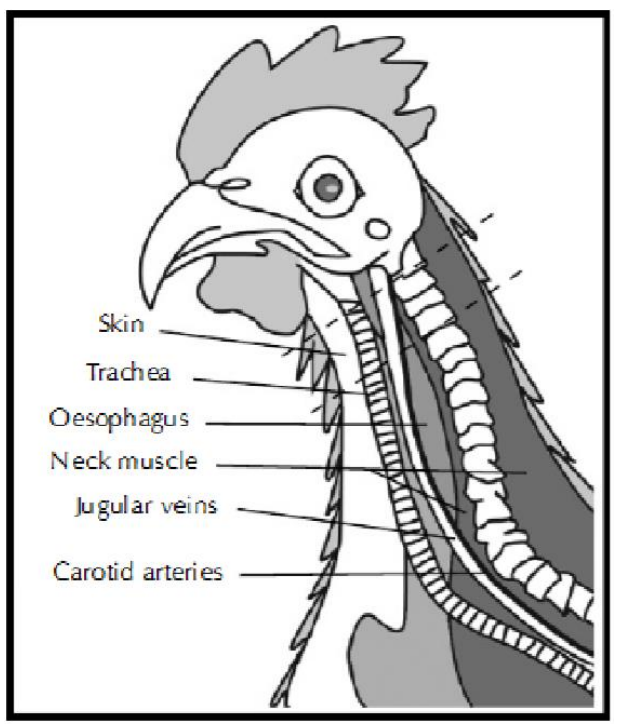

Fig. 2 - Anatomy of chicken's neck (Selamat, 2011)

\section{Methodology}

In the SYCUT system, a slaughtered chicken will pass through different stages of the slaughtering process including the vision inspection system which will detect the esophagus thus classifying the status of the chicken whether it is halal or non-halal. The vision inspection system consists of two stages which the first one being the detection of the esophagus. If the esophagus is not detected, then the chicken will be considered as non-halal and 
removed from the conveyor. The next stage would be to verify the halal status of the chicken with the detected esophagus whether it is halal or not. In this paper, only the first part of the vision inspection system is described.

The first stage of the SYCUT system process is the showering stage, where the chicken is showered with water in order to remove the blood for better inspection or image acquisition. Then image of the cut will be acquired using both the video and also still images. These images will be processed by the vision inspection system software to determine whether the esophagus is detected or not. Finally, the labelling stage marks the non-halal chicken by means of a colour gun. The operator will manually remove the labelled non-halal chicken.

There are two main parts of vision inspection system which are image acquisition and esophagus detection. Two sensors installed to detect the presence of the chicken and indication for the camera to capture images of the chicken. In order to increase the overall image quality, an image pre-processing technique is used, in a way of reducing the noise and standardize the image contrast. Then, image segmentation is done to separate the background from the foreground. This is important to reduce false positive esophagus samples. Finally, the detection stage will locate the esophagus in the foreground image. The summarized components can be seen as in Fig. 3.

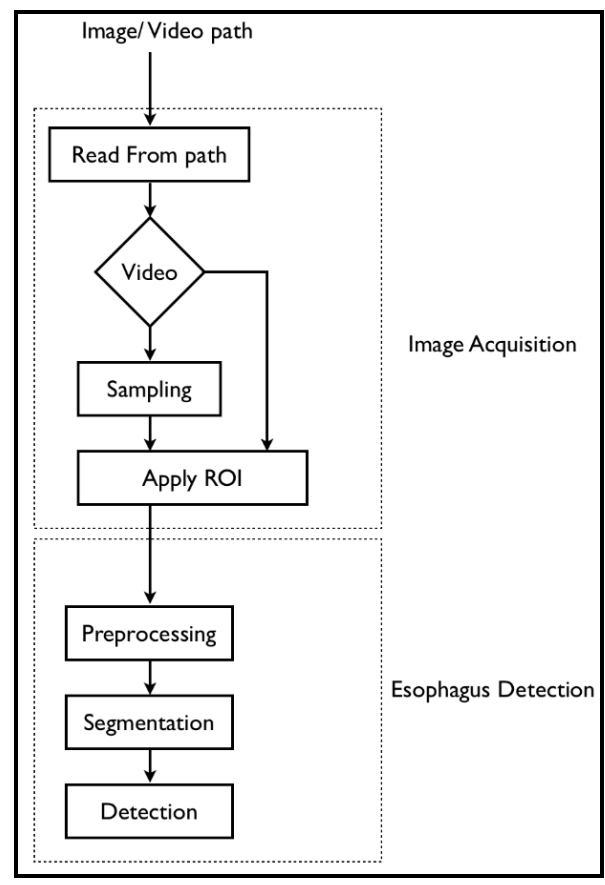

Fig. 3 - Major components used in SVIT (Arfa, 2015)

\subsection{Image Acquisition}

This stage accepts still image or video image as input. It will perform sampling in the case of video image. The output of image acquisition is a still image of the chicken. The last stage of image acquisition is the cropped image by the predefined Region of Interest (ROI).

The camera used to capture the images is a Panasonic Network Camera. The camera has the capability to integrate with our developed software. Its functionality such as the ability to autofocus, pan, tilt and zoom are important in order to capture a good image of the chicken's neck for detection.

\subsection{Detection}

This stage finds the chicken's esophagus position in the acquired image. There is a chain of stages to perform detection which are pre-processing, chicken segmentation and esophagus detection.

\subsubsection{Pre-Processing}

Low pass filter (LBF) is used to suppress the noise from the raw acquired image (Makandar, A., \& Bhagirathi, H., 2015). LBF passes low frequency pixel variation while attenuate high frequency variation. The noise from images are often due to analog to digital conversion especially in poor illumination environments. Fig. 4 shows the images before and after applying filter. 

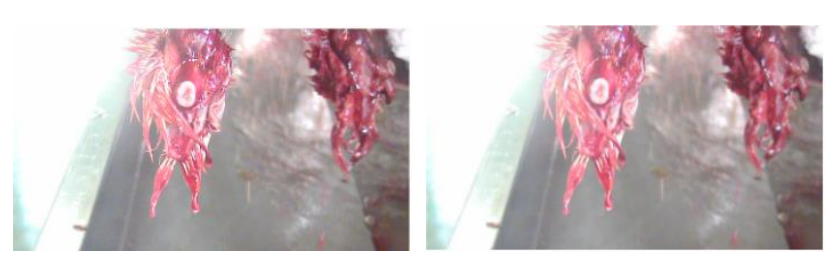

Fig. 4 - Original image (left) and image after LPF filter (right) (Arfa, 2015)

\subsubsection{Segmentation}

The slaughtered chicken and background are separated in this stage. The RGB color space image is converted to $\mathrm{YCbCr}$ color space. The color space in YCbCr color space is encoded in terms of luma (Y) and two blue-difference and red-difference chrominance components $(\mathrm{Cb}$ and $\mathrm{Cr})($

Fig. 5).

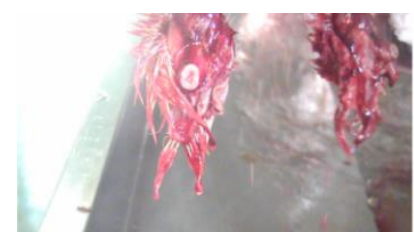

(a)

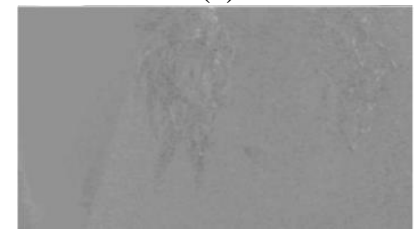

(c)

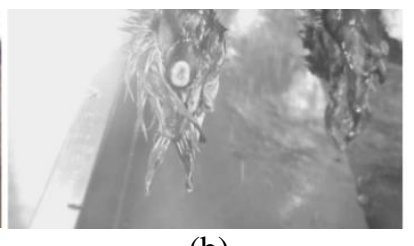

(b)

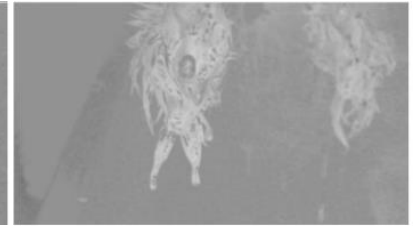

(d)

Fig. 5 - Decomposition of filter image (a) to (b) Y, (c) Cb, (d) Cr (Arfa, 2015)

Then, thresholding is applied to segment the slaughtered chicken from the background. Determining a fixed threshold value is not possible due to changes of imaging system, lighting ambiance and camera settings. Therefore, an Otsu thresholding technique is used to dynamically determine the threshold value based on each image's statistics (Birdal, 2009).

Next, some unwanted holes in the foreground and background are fixed by using morphological technique to erode the holes from background and dilate the holes in foreground. After we get a mask which classifies the foreground and background by a binary image, we multiply the mask to each R, G, and B channel element-wise. This then results in the final segmented image of separated foreground and background (Fig. 6).

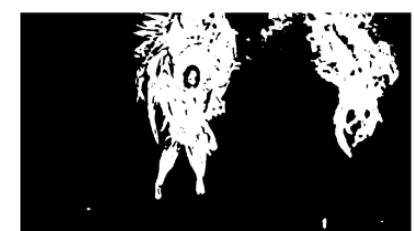

(a)
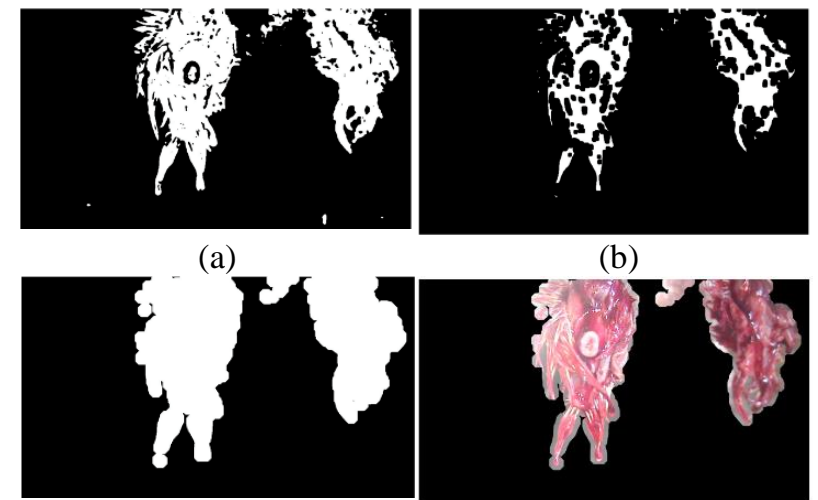

(c) (b)

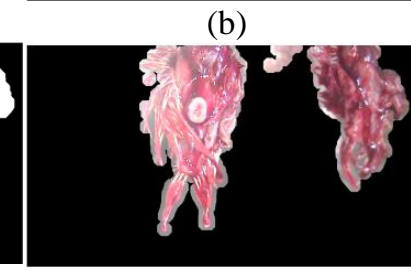

(d)

Fig. 6 - (a) Otsu thresholding, (b) Erosion, (c) Dilation, (d) final segmented image (Arfa, 2015) 


\subsubsection{Esophagus Detection}

The detection stage is created by using the idea from Viola and Jones Object Detection Framework (Viola \& Jones, 2001). This framework is chosen due to its fast detection rate for real-time application. The three ideas introduced by (Viola \& Jones, 2001) in their object detection framework are the integral image to compute haar features efficiently, boosting learning for weak classifier and the attentional cascade structure for fast negative rejection (Freund, Y., \& Schapire, R. E., 1997). The following sub-sections describe framework of Viola \& Jones (2001) briefly and its implementation in SYCUT as reported in "Finding the Optimal Value of Minimum Neighbors Parameter in Viola \& Jones Object Detection Framework Through Empirical Analysis: Application to Esophagus Detection in Syariah Compliance Automatic Chicken Processing System (SYCUT)" (Yusof \& Yunus).

\subsubsection{Features and Integral Image}

A set of features that are reminiscent of Haar Basis functions (Fig. 7) are used by Viola \& Jones (2001). These rectangle features are quite large and therefore integral image representation of images are introduced (Fig. 8). In general, the following equation can be used to compute integral image at location $\mathrm{x}, \mathrm{y}$ :

Where:

$$
i \bar{u}(x, y)=\sum_{x^{n} \leq x y s y^{i}} i\left(x^{n}, y^{n}\right)
$$

$$
\begin{aligned}
& \tilde{i}(x, y) \text { - integral image } \\
& \tilde{i}(x, y) \text { - original image }
\end{aligned}
$$

By using the following recurrences, the integral image can be computed in one pass over the original image as shown by VJ:

$$
\begin{aligned}
s(x, y) & =s(x, y-1)+i(x, y) \\
i i(x, y) & =i i(x-1, y)+s(x, y)
\end{aligned}
$$

where $s\left(x_{y} y\right)$ is the cumulative row sum, $s\left(x_{x}-1\right)=0$ and $i i(-1, y)=0$.

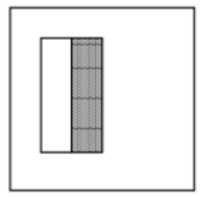

A

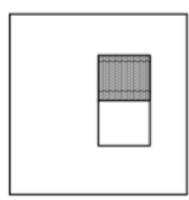

B

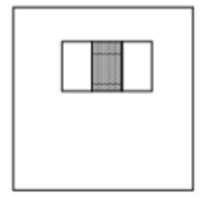

C

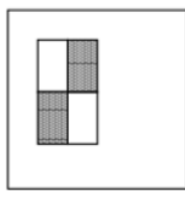

D

Fig. 7 - Examples of rectangle features. $A$ and $B$ are two-rectangle features while $C$ is a three-rectangle feature and $D$ is a four-rectangle feature. Adapted from Viola \& Jones (2001).

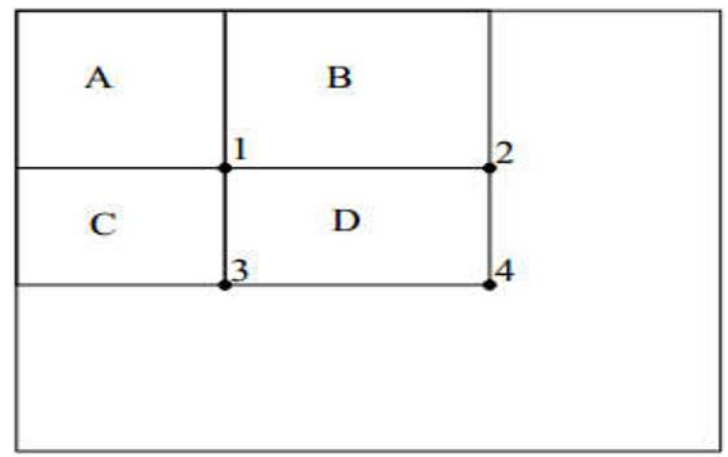

Fig. 8 - Image is divided into 4 regions. Region D can be computed by summing region D and A, and subtracting the summation of region $B$ and C (4+1-(2+3)). Adapted from Viola \& Jones (2001).

\subsubsection{Feature selection}

A number of important features are selected by using adaptive boosting algorithm (Adaboost) (Freund, Y., \& Schapire, R. E., 1997). These features are obtained through a small modification on existing Adaboost procedure by 
constraining the weak learner (Tieu, K., \& Viola, P., 2004). This way, each weak learner can depend on only single features. The final classifier is the linear combination of these weak learners which forms the strong classifier. The details of this algorithm can be referred at the paper by Viola \& Jones (2001).

\subsubsection{Attentional Cascade}

A method of degeneration decision tree (cascade) is used to limit the number of features computed per sub window. Decision nodes for the tree are built by using several stages as shown in Fig. 9. The sub-window is rejected if any stage in the tree rejects it.

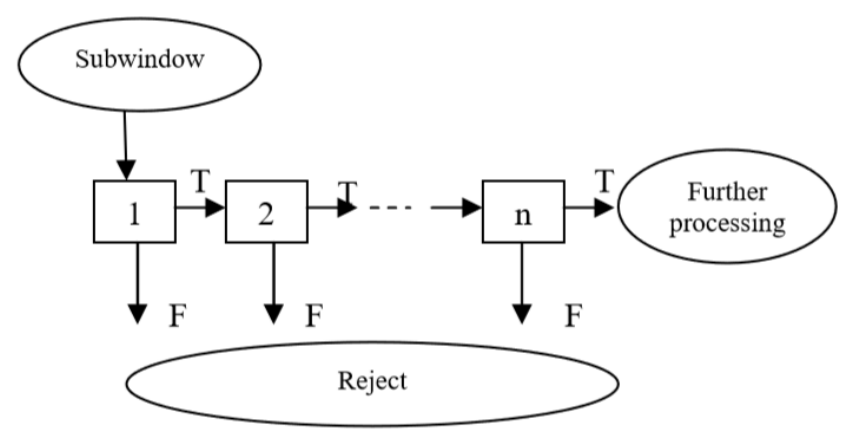

Fig. 9 - Attentional cascade. Adapted from Viola \& Jones (2001).

Each stage of classifier is trained to detect nearly every objects of interest while rejecting a small number of nonobject samples Framework (Viola \& Jones, 2001). Specifically, according to our case, each stage is designed to reject majority of the non-esophagus samples while falsely eliminating a few esophagus samples.

Implementation of the Viola and Jones training algorithm is done using OpenCV modules such as CvCascadeClassifier class and object detection testing in CascadeClassifier class ("Haar Feature-based Cascade Classifier for Object Detection", n.d.). The function DetectMultiScale in CascadeClassifier class can be used to load the trained cascade classifier and detect the object. A list of parameters as shown in Table 1 need to be set by the user in this function.

Table 1 - DetectMultiScale function parameters and its description (Yusof \& Yunus)

\begin{tabular}{|c|c|}
\hline Parameter & Description \\
\hline 1. image & $\begin{array}{l}\text { Matrix of the type } \mathrm{CV}_{-} 8 \mathrm{U} \\
\text { containing an image where } \\
\text { objects are detected. }\end{array}$ \\
\hline 2. $\quad$ objects & $\begin{array}{l}\text { Vector of rectangles where } \\
\text { each rectangle contains the } \\
\text { detected object. }\end{array}$ \\
\hline 3. scaleFactor & $\begin{array}{l}\text { Parameter specifying how } \\
\text { much the image size is } \\
\text { reduced at each image scale. }\end{array}$ \\
\hline 4. minNeighbors & $\begin{array}{lrr}\text { Parameter } & \text { specifying } & \text { how } \\
\text { many neighbors } & \text { each } \\
\text { candidate rectangle should } \\
\text { have to retain it. }\end{array}$ \\
\hline 5. flags & $\begin{array}{l}\text { Parameter with the same } \\
\text { meaning for an old cascade as } \\
\text { in the function } \\
\text { cvHaarDetectObjects. It is not } \\
\text { used for a new cascade. }\end{array}$ \\
\hline 6. $\operatorname{minSize}$ & $\begin{array}{l}\text { Minimum possible object } \\
\text { size. Objects smaller than that } \\
\text { are ignored. }\end{array}$ \\
\hline 7. maxSize & $\begin{array}{l}\text { Maximum possible object } \\
\text { size. Objects larger than that } \\
\text { are ignored. }\end{array}$ \\
\hline
\end{tabular}


One of the parameters needed to be set is the minimum neighbors parameter. If the value is set too low, then the algorithm will produce a lot of false positive and if the value is set too high, then the object of interest may not be detected.

The cascade classifier was trained by using about 8000 positive images of chicken esophagus from two different sites (Az-Zain and 3P) and more than 9000 negative images. It is trained by using an OpenCV function called "opencv_traincascade" using Haar features.

Through the evaluation of the detection algorithm, it is found that the optimal value for neighbors parameter value in object detection function is 9 (without ROI) and 7 (with ROI).

After the possible positions of esophagus are determined by Haar Classifier (Fig. 10 (a)), verification is done using Support Vector Machine (SVM) (Osuna, E., Freund, R., \& Girosi, F., 1997). Each image of candidate esophagus is given to SVM classifier and are labelled as "True" or "False". The final detected esophagus is seen as in Fig. 10 (b).

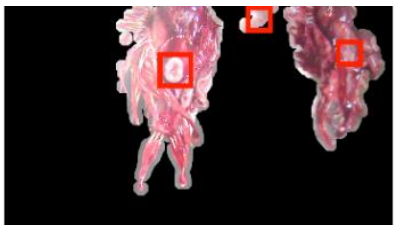

(a)

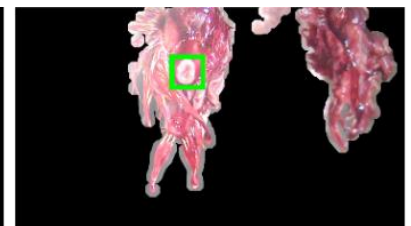

(b)

Fig. 10 - Finding esophagus in real time with approximately 50ms elapsed time (Arfa, 2015)

\section{Experimental Result and Discussion}

This section explains the experimental result and analysis. A database of sample images is formed in order to conduct these experiments. The sample images are taken at two slaughter houses namely the Az-Zain in Semenyih and 3P in Johor Bahru. The total sample images are 265 and 1800 for Az-Zain and 3P sites respectively (Arfa, 2015).

There are three primary goals to the experiments done. The first goal which is obtaining the overall accuracy of current system on both sites gives us a general idea of how good the system works. The second goal being the evaluation of the system in algorithmic perspective shows how efficient and robust the system performs on different sites and different lighting situations. The third goal which is identifying the limitations caused by external environmental factors allow us to better understand the problems faced in factory environments and how we can overcome them.

\subsection{Overall Performance}

Assuming that most chickens are slaughtered the halal way, there should be a detected esophagus on each chicken in an image. Based on this criterion, an experiment is done to measure the overall performance of the systems in both sites. The results tell us how efficient the current system is.

A 0-1 loss function is used where if the system does not detect the esophagus, an error of 1 is added to overall error (Grosse, R., n.d.)Error! Reference source not found.. The overall performance can be seen from Table 2 below. If the overall performance is $81.8 \%$, then it means that out of 100 chickens, the system is able to detect almost 82 of them being halal despite any other environmental factor.

It is concluded that the inefficiency of the overall system can be caused by the esophagus detection stage or the image acquisition stage. There is a big gap between Az-Zain and 3P sites. Experiments for the individual stages help us to identify the problems further.

Table 2 - Overall Accuracy Based on The Assumption That All the Chickens on Conveyor Are Halal (Arfa, 2015)

\begin{tabular}{lcc}
\hline Sites & Az-Zain & 3P \\
\hline Overall Performance & $81.8 \%$ & $55.5 \%$ \\
\hline
\end{tabular}




\subsection{Performance of Esophagus Detection}

This section aims to evaluate the system's performance without consideration of any external factors. The set of images are manually chosen to make sure that those images contain visible esophagus which a human expert can detect. An ideal detection system should be able to detect the esophagus in this set of images and does not detect nonesophagus area as esophagus.

The three terminologies used in the result analysis are False Positive (FP), True Positive (TP) and False Negative (FN). Below is the explanation of these terminologies based on the project's case:

1. FP: System mistakenly detects a non-esophagus area as esophagus.

2. TP: System correctly determines an esophagus in an image.

3. FN: System fail to detect an esophagus.

The detection system experimental result is presented by Detection Rate (DR) and False Positive Rate (FPR).

$\mathrm{DR}$ is the ratio between number of times an esophagus is detected correctly by the system to the number of esophagus determined by human expert. The following equation captured the relation between TP, FN and DR (Kumar, G., 2014):

$$
D R=\frac{T P}{T P+F N}
$$

FPR is the ratio between false positive alarm and total number of sub-windows (Fawcett, 2006):

$$
F P R=\frac{F P}{F P+T N}
$$

An ideal detection system should have DR of $100 \%$ and zero FPR. The result of the experiment is recorded in the Table 3 below. The detection stage is able to perform well under reasonable variation of illumination and pose.

Table 3 - Esophagus Detection Accuracy (Arfa, 2015)

\begin{tabular}{lcc}
\hline Sites & Az-Zain & 3P \\
\hline Detection Rate & $95.6 \%$ & $93.5 \%$ \\
\hline False Positive Rate & 1 in 200,000 & 1 in 250,000 \\
\hline
\end{tabular}

\subsection{Performance of Image Acquisition}

Next, the image acquisition stage which is one of the main stages of the vision inspection system was tested for its performance. An ideal image acquisition system should have $0 \%$ invisibility rate. The test shown that the esophagus invisibility rate which mainly caused by pose variation and occlusion affects the detection performance. The results in the Table 4 below shows that $40.7 \%$ of the images taken in $3 \mathrm{P}$ site has no visible esophagus to be detected by a human agent.

Table 4 - Invisibility Rate (Arfa, 2015)

\begin{tabular}{lcc}
\hline Sites & Az-Zain & 3P \\
\hline Invisibility Rate (\%) & $14.4 \%$ & $40.7 \%$ \\
\hline
\end{tabular}

In this case of invisibility, the reasons to invisible esophagus in those images and their percentage are as Table 5 below.

Table 5 - Reason of Invisible Esophagus (Arfa, 2015)

\begin{tabular}{lcc}
\hline Sites & Az-Zain & 3P \\
\hline Pose Variation & $79.4 \%$ & $62.9 \%$ \\
\hline Occlusion & $16.2 \%$ & $37.1 \%$ \\
\hline
\end{tabular}


The experimental results show that the detection performance is promising but the image acquisition performance proved to correspond to most of the failures of SVIT. There were some experimental changes done to tackle the problems and decrease error. The proposed solutions are in regards to algorithmic and physical structure perspective.

One of the solutions is by adding another light source to the lighting system. The amount of light hitting the chicken is controlled to about 60-70 foot-candles. The new light source is placed at the opposite direction of the previous lighting system to balance shadows. The invisibility rate was decreased by $13.6 \%$ by using this lighting system while the overall system accuracy increased by $11.9 \%$.

Next, the proposed solution for overall system's performance is to use video processing. This is proposed in accordance to the idea that the system is able to decide better with 60 different images rather than just a few images of a certain chicken. The problems like pose variation or chicken orientation is likely to have more chance of esophagus detection in case where the chicken head keeps turning around on the conveyer. Other than that, there is also a need for tracking the individual chickens and assigning an ID to each chicken.

Additionally, the next proposed solution is to use multiple camera to solve the pose of chicken's neck problem. Adding another camera with different angle of view can capture the chicken's neck in another angle, with hopes that the esophagus can be seen. On the other hand, adding the number of cameras doubles the processing time and increase the system's complexity. Experiment done by adding another camera on the right side or left side yield an increase of $2 \%$ or $20 \%$ improvement in the overall system performance respectively.

Improving the image acquisition performance is a challenging problem and thus these and other possible solutions should be analysed and experimented further to help increase overall accuracy of the system.

\section{Conclusion}

This paper presented the technical description and performance analysis of SYCUT vision inspection system. The detection of the esophagus is developed in order to identify whether the chicken is slaughtered properly according to syariah. The two main parts of the vision inspection system are image acquisition and esophagus detection. Various methods and techniques are used in order to improve the accuracy of the system in which one of them is the Viola and Jones object detection framework.

The experiments and analysis are described where some of the challenges faced by the system are found. The accuracy is mostly affected by the lack of illumination, noises from splattering of blood and occlusion from shadows and feathers. The improvements and possible solutions are in terms of the change in lighting control, the use of video processing method instead of images and implementing multiple cameras on the site. Future improvements on the system's accuracy are in progress.

\section{Acknowledgement}

The authors gratefully acknowledge the support given to this project by the Malaysian government under the TechnoFund grant. Authors would also like to express appreciation to Mumtaz Meat \& Marine Food Sdn. Bhd. (AzZain) and Pusat Penyembelihan \& Pemprosesan Ayam (3P) Sdn. Bhd. for the cooperation given while conducting experiment and research at their sites.

\section{References}

[1] Selamat, H. (2011). Technofund Technical Report on Syariah-Compliance Automated Chicken Processing Machine for Small and Medium Sized Industries. Kuala Lumpur: UTM, Center for Artificial Intelligence and Robotics (CAIRO).

[2] Omar, E. N., \& Jaafar, H. S. (2011). Halal supply chain in the food industry - A conceptual model. 2011 IEEE Symposium on Business, Engineering and Industrial Applications (ISBEIA) (pp. 384-389). Langkawi, Malaysia: IEEE. doi:https://doi.org/10.1109/ISBEIA.2011.6088842

[3] Mohd. Yunus, A., Wan Chik, W., \& Mohamad, M. (2010). The Concept of Halalan Tayyiba and Its Application in Products Marketing : A Case Study at Sabasun HyperRuncit Kuala Terengganu, Malaysia. International Journal of Business and Social Science., 1, 239 - 248.

[4] al-Qaradawi, S. Y. (2013). The Islamic Manner of Slaughtering. In The Lawful and The Prohibited in Islam. Islamic Book Trust.

[5] Yusof, F. M. (2006). The Modern Methods of Animal's Slaughtering in Malaysia: An Islamic Perspective. Universiti Sains Islam Malaysia. Retrieved from http://ddms.usim.edu.my:80/jspui/handle/123456789/5009

[6] Chand, M. U. (1995). Halal and haram : the prohibited and the permitted foods and drinks according to Jewish, Christian and Muslim scriptures. Kuala Lumpur: A.S. Noordeen. 
[7] Department of Islamic Development Malaysia (JAKIM). (2011). Malaysian Protocol for the Halal Meat \& Poultry Production.

[8] Arfa, R. (2015). SYCUT Vision Inspection Technology (SVIT) Technical Report. Kuala Lumpur.

[9] Makandar, A., \& Bhagirathi, H. (2015). Image Enhancement Techniques Using Highpass and Lowpass Filters. International Journal of Computer Applications, 109(14), 12-15.

[10] Birdal, T. (20 July, 2009). Famous Otsu Thresholding in C\#. Retrieved from Code Project: https://www.codeproject.com/Articles/38319/Famous-Otsu-Thresholding-in-C

[11] Viola, P., \& Jones, M. (2001). Rapid object detection using a boosted cascade of simple features. Proceedings of the 2001 IEEE Computer Society Conference on Computer Vision and Pattern Recognition. CVPR 2001 (pp. I-I). Kauai, HI, USA: IEEE. doi:https://doi.org/10.1109/CVPR.2001.990517

[12] Freund, Y., \& Schapire, R. E. (1997). A Decision-Theoretic Generalization of On-Line Learning and an Application to Boosting. Journal of Computer and System Sciences, 55(1), 119-139. doi:https://doi.org/10.1006/jcss.1997.1504

[13] Yusof, R., \& Yunus, R. (n.d.). Finding the Optimal Value of Minimum Neighbors Parameter in Viola \& Jones Object Detection Framework Through Empirical Analysis: Application to Esophagus Detection in Syariah Compliance Automatic Chicken Processing System (SYCUT).

[14] Tieu, K., \& Viola, P. (2004). Boosting Image Retrieval. International Journal of Computer Vision - Special Issue on Content-Based Image Retrieval, 56(1-2), 17-36. doi:https://doi.org/10.1023/B:VISI.0000004830.93820.78

[15] Haar Feature-based Cascade Classifier for Object Detection. (n.d.). Retrieved from OpenCV 2.4.13.7 documentation: https://docs.opencv.org/2.4/modules/objdetect/doc/cascade_classification.html\#cascadeclassifiercascadeclassifier

[16] Osuna, E., Freund, R., \& Girosi, F. (1997). Training Support Vector Machines: an Application to Face Detection. Proceedings of the 1997 Conference on Computer Vision and Pattern Recognition (CVPR '97 (p. 130). USA: IEEE.

[17] Arfa, R. (2015). SYCUT Vision Inspection Technology (SVIT) Analysis and Future Works.

[18] Grosse, R. (n.d.). Lecture 4: Training a Classifier. Retrieved from Computer Science University of Toronto: http://www.cs.toronto.edu/ rgrosse/courses/csc321_2017/readings/L04\%20Training\%20a\%20Classifier.pdf

[19] Kumar, G. (2014). Evaluation Metrics for Intrusion Detection Systems - A Study. International Journal of Computer Science and Mobile Applications, 2(11), 11-17.

[20] Fawcett, T. (2006). An introduction to ROC analysis. Pattern Recognition Letters, 27(8), 861-874. doi:https://doi.org/10.1016/j.patrec.2005.10.010 